\title{
A competence-based industrial learning approach for factories of the future
}

\author{
Dimitris Kiritsis • Ahmed Bufardi • \\ Dimitris Mavrikios • Thomas Knothe • \\ Hadrien Szigeti • Anirban Majumdar
}

Published online: 17 January 2013

(C) Springer Science+Business Media New York 2013

\begin{abstract}
Manufacturing industry can improve its competitiveness through innovation and technological excellence, and appropriate Industrial Learning can help to achieve this goal through allowing the manufacturing workforce to acquire new skills related to the advanced developments in information and communication technologies. This raises the need for new Industrial Learning tools and methods from the viewpoint of learning content, learning processes, and delivery mechanisms. In this paper, we present a generic competence-based approach for Industrial Learning developed in the framework of ActionPlanT project. The approach is composed of (i) an Industrial Learning model which serves to represent and understand competence-based learning, and (ii) a methodology which implements through a number of steps the Industrial Learning actions defined using the Industrial Learning model in industrial organisations. Both the model and the methodology
\end{abstract}

D. Kiritsis $(\bowtie)$

STI-IGM-LICP, EPFL, ME A1 396, Station 9, 1015 Lausanne, Switzerland e-mail: dimitris.kiritsis@epfl.ch

A. Bufardi

STI-IGM-LICP, EPFL, ME A1 403, Station 9, 1015 Lausanne, Switzerland

e-mail: ahmed.bufardi@epfl.ch

D. Mavrikios

Laboratory for Manufacturing Systems and Automation, University of Patras, Patras 26500, Greece e-mail: mavrik@1ms.mech.upatras.gr

T. Knothe

Fraunhofer Institut für Produktionsanlagen und Konstruktionstechnik, Pascalstasse 8-9,

10587 Berlin, Germany

e-mail: Thomas.Knothe@ipk.fraunhofer.de

H. Szigeti

Dassault Systemes, 10 rue Marcel Dassault, CS 40501, 78946 Velizy Villacoublay Cedex, France e-mail: Hadrien.SZIGETI@3ds.com

A. Majumdar

SAP research center, Chemnitzer Strasse 48, 01187 Dresden, Germany

e-mail: anirban.majumdar@sap.com 
are presented in details. A metrics-based method for evaluating the implementation of the learning actions defined using the approach is also described.

Keywords Industrial learning - Competence development - ICT for manufacturing · Factories of the future

\section{Introduction}

In this paper, we present a generic competence-based approach for Industrial Learning developed in the framework of the FP7 FoF project ActionPlanT: European Forum for ICT in Factories of the Future. The research project ActionPlanT has been co-funded by the European Commission under the Private-Public Partnership (PPP) "Factories of the Future" initiative of the Seventh Framework Programme (FP7) for research and technological development (Grant Agreement Number 258617). The main goal of ActionPlanT was to develop a vision on the short, medium, and long term role of Information and Communication Technology (ICT) in the European manufacturing industry. The research project started in June 2010 and closed in July 2012.

For Europe to hold on to its global leadership and excellence in manufacturing, it is imperative that improvements at both the technological and the awareness level are made for ICT-enabled manufacturing processes. ActionPlanT was set out to address the improvement in this respect of the short, medium and long term role of ICT in the manufacturing industry. On a more holistic level, ActionPlanT results outline the vision of the future role of ICT in manufacturing for the European Commission's next research framework program Horizon2020. Moreover, a concept of disseminating knowledge and future requirements is explored through a well-established platform consisting of manufacturing and ICT experts from both academia and industry.

In summary, the two main activities of ActionPlanT were:

- Establishing an ICT-enabled manufacturing vision for use cases and services of the future using this analysis as a basis. This vision will pave the way for a detailed roadmap which will prioritize and schedule most promising topics for the upcoming work program for Research and Innovation of Framework Programme (Horizon2020) ;

- Developing and validating a concept for Industrial Learning (IL), extensively piloted via Industrial Learning Pilot Events (ILPEs) and workshops amongst stakeholders in industry, academia, and the European technology platforms alike.

These two parallel work streams "Vision \& Roadmap" and "Awareness \& Industrial Learning" of ActionPlanT project are depicted in Fig. 1.

The approach presented in this paper relates to the second work stream "Awareness \& Industrial Learning".

\section{Rationale for industrial learning}

Promoting excellence in manufacturing emerges as a strategic goal in the years to come, both for industry and society. 
Manufacturing education is expected to be a major driver to achieving this goal. To respond to this role, manufacturing education should follow a new approach to prepare industry for the next-generation innovation and support its growth (Manufuture 2006). More specifically, it should focus on:

- promoting synergy between the academia stakeholders and industry; the comprehension of the needs of the manufacturing industry for training and education, the joint definition of the content, the pedagogic approach and the delivery mechanisms for future curricula, as well as the integration of research and innovation with education and training activities, are considered as the main priorities;

- developing the ICT for manufacturing skills required by the manufacturing labour force to face new professional needs; the adaptation of educational content and its delivery mechanisms to the new requirements of ICT-based manufacturing, the provision of integrated engineering competencies, including a variety of soft skills, and the promotion of innovation and entrepreneurship spirits, are considered as major priorities.

In order to achieve these objectives, manufacturing education has to address several challenges in the years to come. As far as the IL aspect of manufacturing education is concerned, some major challenges are discussed hereafter.

New skills are required by the future generations of "knowledge workers". To that direction, an adaptation of the educational content and its delivery mechanisms to the new requirements of knowledge-based manufacturing is required. Manufacturing strategy with focus on digital business, extended production and virtual enterprises should be greatly considered. On the other hand, there is a growing need for expanding the technological aspect of education, with an extension to the 'soft skills'.

The development of educational curricula has not kept pace with the growing complexity of industry, technology and economy. Moreover, research outcomes of educational institutions are typically presented to the scientific community without

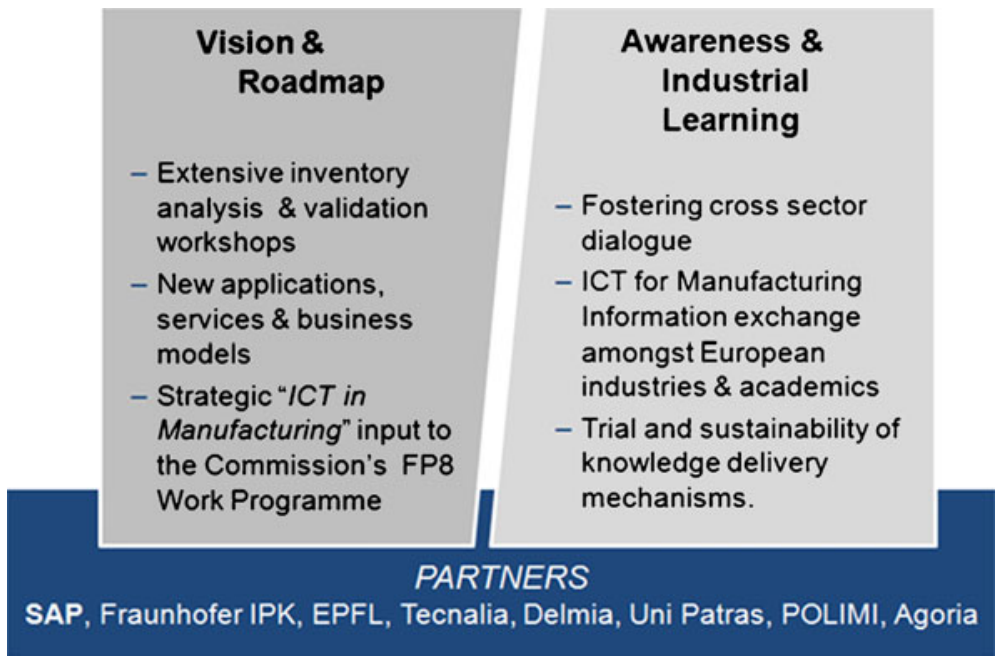

Fig. 1 ActionPlanT Parallel Work streams 
being directly accessible to industry. Within this context, it is difficult for industry to comprehend and to adapt to the technological advances in a direct way.

In the industrial context knowledge is generated by Universities and Research institutes and implemented in Industries as illustrated by the Knowledge Triangle concept in Fig. 2 (Westkämper 2008). ActionPlanT covers the area within the marked (green) border. This figure illustrates the sources of knowledge to be transferred to Industry using the ActionPlanT IL Model.

The need for integrating the cornerstones of the knowledge triangle (Fig. 2) into a single framework for supporting manufacturing education, has given rise to a number of learning paradigms and mechanisms.

\section{State of the art and gap analyses}

The state of the art analysis which has been done based on a large number of references among which Batista et al. (2009); CEDEFOP (2009); Chen and Chen (2004); Chi and Speeding (2007); Chryssolouris (2005, 2006); Chryssolouris and Mavrikios (2005); Chryssolouris and Mourtzis (2008); Chryssolouris et al. (2002, 2006); Ellstrom and Kock (2009); Folstad (2008); Hamade et al. (2011); Hauge et al. (2006); IMS2020, Kramer et Azadivar (2002); Lamancusa et al. (2001); Marquardt et al. (2011); Mavrikios et al. (2005); Mayes and Freitas (2005); Mirehei (2009); O'Sullivan et al. (2009); OECD (2004); Paci and Lalle (2008); Rolstadås and Dolinšek (2006); Rolstadås and Hussein (2002); Schaf et al. (2009); SME (2010); Wong et al. (2010); Yaldiz (2010); Zimmermann (2010) reveals that the existing learning methodologies suffer from the following weaknesses:

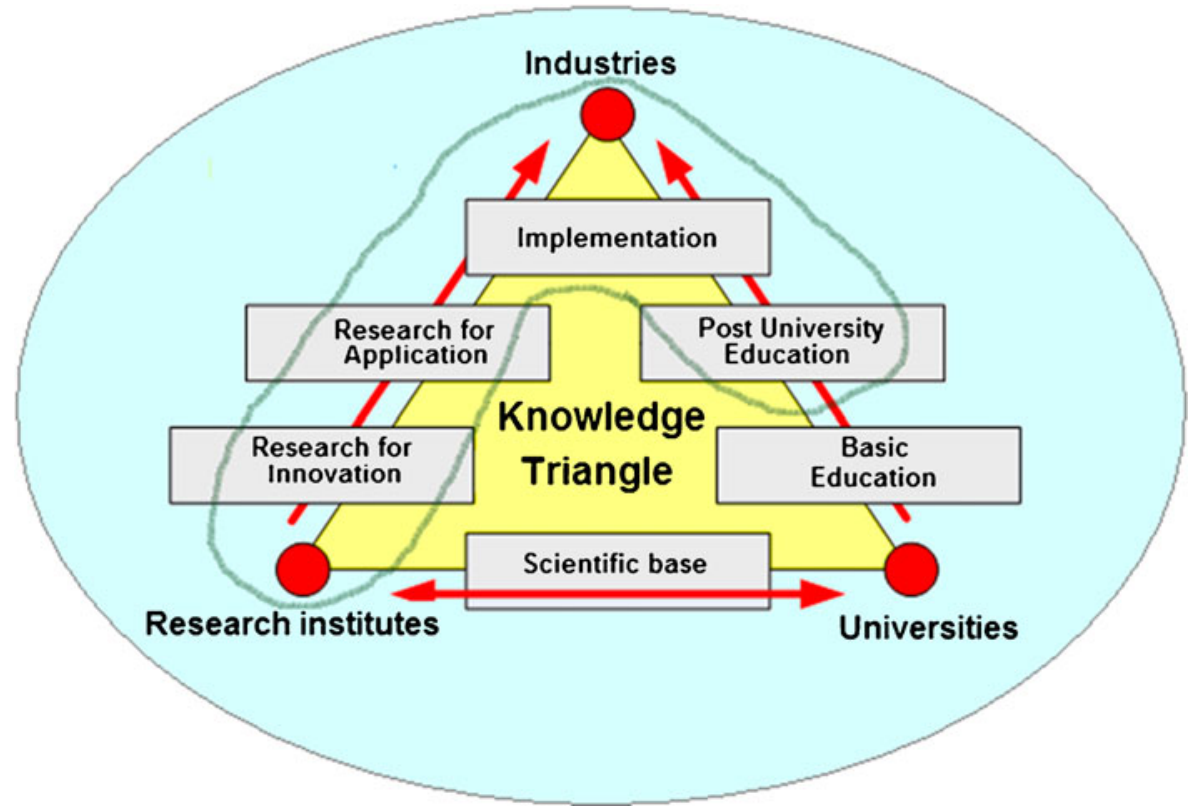

Fig. 2 The Knowledge Triangle in industrial education (Westkämper) 
- The definition of professional competencies is based on company internal needs analysis which excludes new ICT for manufacturing competencies defined from knowledge assets created from other sources such as research \& innovation projects, best practices, etc.;

- Most of existing learning methodologies focus on the transfer of "mature" knowledge (e.g., knowledge developed several years ago);

- The existing learning approaches are type-specific (e.g., vocational training, technology transfer, etc.) and vary from one type to the other, due to the different learning goals;

- Most of the existing approaches are customized according to the learning content;

- Despite their potentiality for competence development, Living Labs are mainly considered at the R\&D level (e.g. open innovation platforms, exposing test bed applications to the users, etc.) and have not been systematically used for training so far;

- Most of the reported applications of the Teaching Factory paradigm focus on academic training rather than on IL;

- The emerging ICT-based learning formats, such as collaborative learning environments, game-based learning, virtual reality environments, etc. appear so far only in prototype software applications or highly specialized applications addressing a narrow range of learning cases.

The ActionPlanT IL approach is developed in a way to fill most of the gaps of existing approaches. Among its main characteristics we can quote:

- Using ActionPlanT IL model, the new professional competencies are created from the recent achievements of research and innovation actions in the domain of "cutting edge" ICTs for manufacturing;

- Based on the "extended" Teaching Factory concept, the ActionPlanT framework suggests the integration of research and IL activities, which brings "cutting edge" knowledge in the learning process;

- The ActionPlanT IL approach that addresses all the cognitive range of IL, from attitude building to competence development is generally applicable and may be adjusted to the needs of each specific learning activity;

- The ActionPlanT IL methodology is generally applicable and consequently it can accommodate learning content from a big range of challenging topics on "ICT for Manufacturing";

- ActionPlanT framework suggests Living Labs as a major tool for competencies development;

- ActionPlanT suggests an extended Teaching Factory concept as a basis for the IL framework;

- The ActionPlanT framework integrates the emerging ICT-based learning formats, such as collaborative learning environments, game-based learning, interactive multi-media training, etc., in a systematic approach that addresses all the cognitive range of IL, from attitude building to competence development;

- The evaluation scheme in the ActionPlanT IL methodology considers relevant sets of metrics for impact measurement of the training activities for: attitude, knowledge, skills and competence. 


\section{Competence based learning}

The ActionPlanT model / methodology provides answers to the following questions:

- What are the cognitive / learning aspects to be addressed by the IL activities on ICTs for manufacturing?

- How should these aspects be addressed, namely how should IL on ICTs for manufacturing be delivered?

\subsection{Competence development in the ActionPlanT framework}

The aim would be to address training needs for a systematic, but also visionary, use and exploitation of knowledge and skills for innovating industrial products and processes. Training would address issues such as understanding of opportunities, combining different pieces of new knowledge and developed skills to solve problems, promoting creativity and innovative spirit, etc.

In this framework, IL actions in ActionPlanT were designed along the three main dimensions: Knowledge, Skills and Attitude:

- Transfer of Knowledge: focus on "Industrial Communities of Practice" using Synchronous (webinars, teaching factory, summer school, etc.) and Asynchronous (e-learning, virtual factory, etc.) learning methods and tools;

- Development of Skills: Focus on dissemination in "Vocational Training" audience;

- Influence of Attitude: Focus on dissemination \& awareness rising in society in general and specific target groups (i.e. high schools).

This is illustrated in Fig. 3.

In the Industrial Learning context we have to do with the development and implementation of new professional competencies created by recent achievements of research and innovation actions in the domain of ICT for Manufacturing.

Fig. 3 Building blocks of the learning process

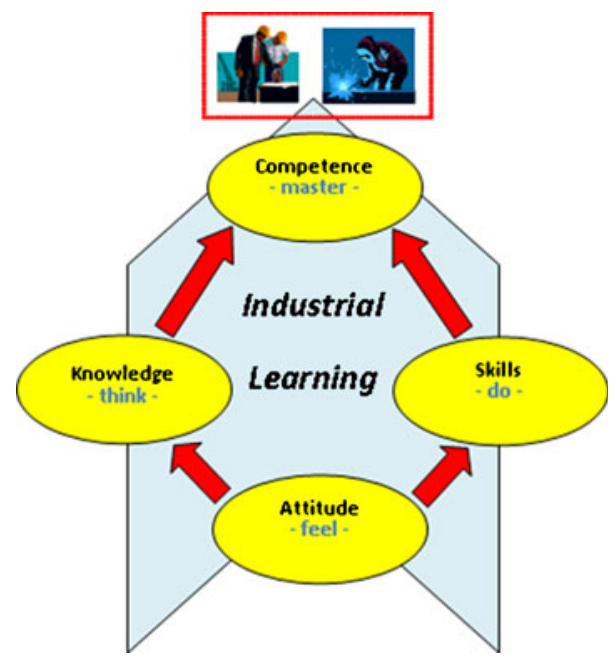




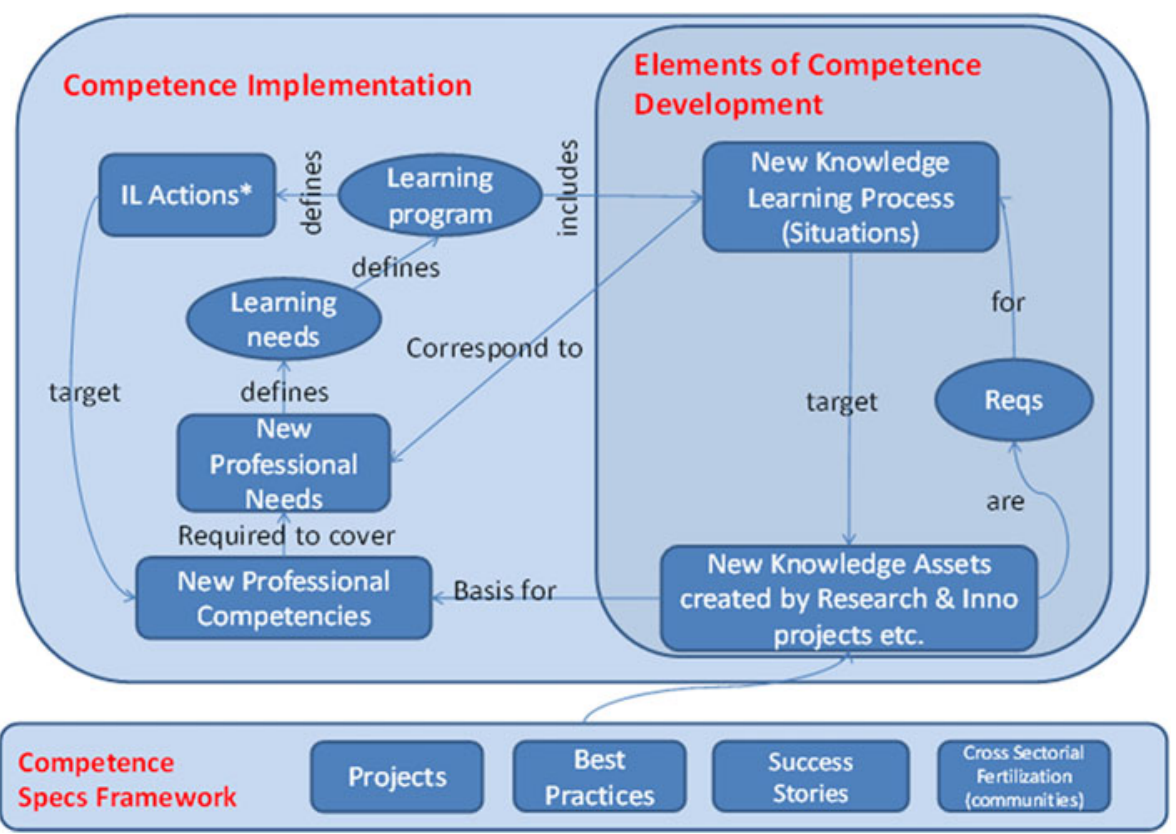

Fig. 4 The ActionPlanT IL Model

\subsection{Building blocks of learning}

In this subsection, we describe the four building blocks of learning: attitude, knowledge, skills and competence (Fig. 3). They are part of the learning process and considered in the learning programs and actions of ActionPlanT IL.

"Attitude" is a hypothetical construct that represents an individual's degree of like or dislike for an item. Attitudes are generally positive or negative views of a person, place, thing, or event.

In the industrial context attitude is the actual perception of manufacturing and related ICT activities by the society in general and the interest that this perception generates for the relevant societal characteristics: job creation, attractiveness of manufacturing activities, will to work in an industrial environment, contribute to "create" something, etc.

"Knowledge" is the outcome of the assimilation of information through learning. Knowledge is the body of facts, principles, theories and practices that is related to a field of work or study.

Knowledge can be seen as the higher level of competence and needs to be continuously updated with new achievements of research and innovation as illustrated in the Knowledge triangle presented in Section 2. The main goal of IL here is the Transfer of Knowledge from research and innovation results to concerned industrial stakeholders.

"Skills" means the ability to apply knowledge and use know-how to complete well defined tasks. Skills may be cognitive (involving the use of logical, intuitive and creative thinking) or practical (involving manual dexterity and the use of methods, materials, and tools). 
Skills are mainly developed through practice and transferred to targeted categories of personnel through appropriate training programs. The main target of IL here is the "Vocational Training" audience and the material would be mature hands-on solutions ready to be introduced into industrial practice.

"Competence" means the proven ability to use knowledge, skills and personal, social and/or methodological abilities. Competence is also described in terms of responsibility and autonomy.

Competences may be considered as the interface between the learning and the innovation processes. As such, the ActionPlanT learning model / methodology addresses competence development as a major requirement.

It is worth noting that ActionPlanT ILPEs have been evaluated on the basis of the improvements made with respect to the four building blocks of learning.

\section{The ActionPlant IL Model}

The ActionPlanT IL Model is adapted from Fürbringer (2010). It is composed of a Competence Specifications Framework (the lower block in Fig. 4) and a Competence Implementation part (the upper block in Fig. 4) which includes a sub-part (inner block) for the identification and definition of Elements of Competence Development (knowledge assets) in a specific sector or, more general, in an Industrial Community of Practice.

\subsection{The competence specifications framework}

In the context of ActionPlanT, the competence specification framework was limited within the scope of "ICT for Manufacturing". The requirements and specifications of the IL actions have been gathered from relevant research projects, best practices etc. and are in line with the first ActionPlanT work stream of Fig. 1.

The competence specifications framework describes the current needs for development of competencies in terms of knowledge, skills, and attitude for a sector or community of practice.

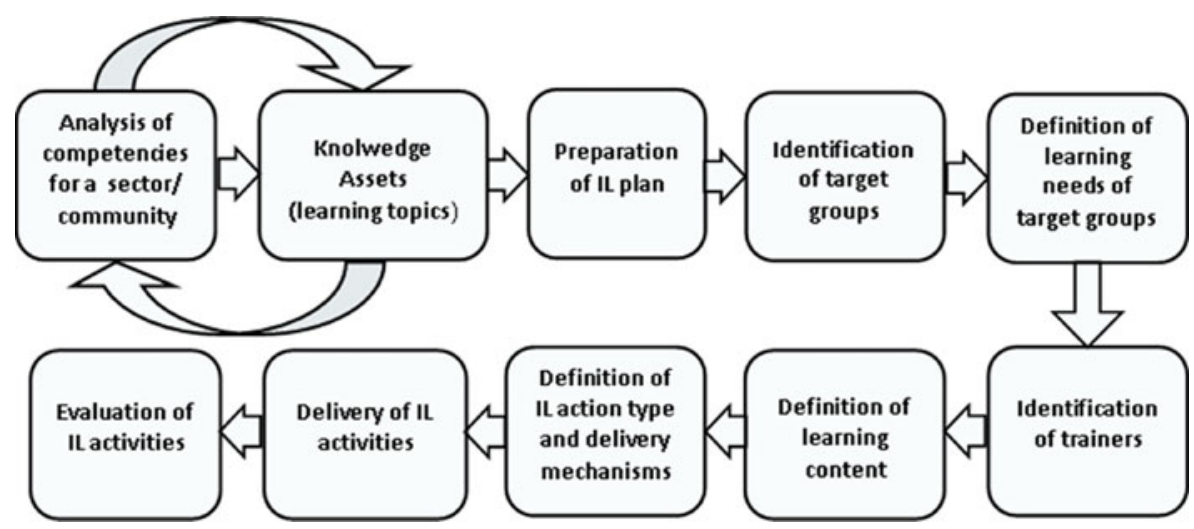

Fig. 5 The ActionPlanT IL methodology 


\subsection{The elements of competence development}

The progress of science and technology creates the so called "knowledge assets" whose transfer in applications in industrial environments create needs and requirements for new professional situations, which are the "Basis for" New Professional Competencies as illustrated by the link in the lower part of the model in Fig. 4. New Professional Competencies cover New Professional Needs, which can be developed through an adequate learning process which is the means to implement and realize the transfer of the defined knowledge assets as illustrated by the link between "New Knowledge Learning Process" and "New Professional Needs" in Fig. 4.

\subsection{Competence implementation}

The knowledge assets and associated learning process that have been defined to cover identified professional needs (and associated competencies) create Learning Needs which are addressed by the training or Human Resources departments of industrial organisations with the design and implementation of Learning Programs which include a well-designed series of IL actions as illustrated by the upper left part of the model in Fig. 4.

\section{The ActionPlant IL methodology}

The ActionPlanT IL methodology aims at implementing through a number of steps the IL actions defined using the ActionPlanT IL model for a specific learning situation. This includes the choice of adequate delivery mechanisms and appropriate evaluation tools. At each step of the methodology, all relevant available techniques including the emerging ones are considered in order to meet the learning styles of the different target audiences and the requirements of the various learning topics.

Unlike traditional IL methodologies which are need-driven meaning that they are designed to respond to specific needs raised by demanding companies, the ActionPlanT IL methodology is opportunity-driven aiming to offer for manufacturing companies an opportunity to develop and implement new professional competencies created by recent achievements of research and innovation actions in the domain of cutting edge ICT for manufacturing.

Exploring the experience developed in training activities of the FP6 PROMISE project (Brintrup and Ranasinghe 2008, Matta et al. 2007) the steps defined in Fig. 5 are the main elements of the methodology to implement IL in industrial communities of practice.

The order of steps in Fig. 5 is specific to the ActionPlanT training case; the order may be different in other training situations.

The different steps of the ActionPlanT IL methodology are described in the following sub-sections.

\subsection{Step 1: ICT for manufacturing competencies analysis}

The ICT for manufacturing competencies analysis is based on the ActionPlanT IL model presented in the previous section. 
In the ActionPlanT IL model new ICT for manufacturing knowledge assets (learning assets) are created from various sources such as research \& innovation projects, best practices, etc. These learning assets provide a basis for the development of new professional competencies in the field of ICT for manufacturing which are useful for companies to develop new knowledge and skills in their manufacturing domains. Indeed, the acquisition by manufacturing companies of the most advanced ICT developed by specialized research institutes and their implementation are often quoted among the main factors to improve competitiveness.

There is an interaction between ICT for manufacturing competencies and ICT for manufacturing knowledge assets. Indeed, the development of an ICT for manufacturing competence requires ICT for manufacturing knowledge asset(s) and an ICT for manufacturing knowledge asset defines one or more ICT for manufacturing competencies.

\subsection{Step 2: ICT for manufacturing topics/modules}

This step is concerned with the identification of the broad topic areas that should be included in the ActionPlanT training program about ICT for manufacturing in order to fulfil the training needs related to the ICT for manufacturing professional competencies.

ActionPlanT framework pursues the direct employment of "cutting-edge" / "fresh" knowledge, produced in recently finished or even still running research projects, in IL activities. That approach can speed up the innovation process.

\subsection{Step 3: Preparation of ActionPlanT IL plan}

The preparation of the ActionPlanT IL plan is based on the analysis of the expertise and the learning infrastructure available for the organization of the IL activity.

In the case of ActionPlanT project, the distribution of learning topics among the different IL actions is based on the expertise and the learning infrastructure available at the organizing ActionPlanT consortium member in order to better use the available resources for ActionPlanT IL learning activities.

\subsection{Step 4: Identification of target groups}

Different audiences are considered in ActionPlanT from different perspectives with regard to their role in ActionPlanT learning process:

- Professional target group including the professional audience at various levels of the manufacturing industry including SMEs as well as among consultancy and relevant service providers.

- Specialized training institutions, professional chambers and their training bodies, etc.

- Academic target group which includes audience from both engineering and vocational training schools.

- Society in general will be considered with possible focused dissemination actions at high school audiences.

For each IL action, the target groups are identified among the audiences mentioned above on the basis of a set of relevant selection criteria such as functional domain, 
manufacturing sector, ICT skills, etc. Nonetheless, the priority will be given to the industrial professionals.

\subsection{Step 5: Definition of learning needs of target groups}

This step deals with the definition of the training needs of the target groups identified in the previous step with respect to the ICT for manufacturing topics considered in Step 2.

Each IL action focuses on one or more learning topics and targets specific audiences. The needs of these audiences relate o the learning topic(s) addressed in the IL action.

A usual technique that is commonly used to address this problem is the "skills matrix" where the target groups are listed in the first column of the matrix and the ICT for manufacturing addressed in the learning topic in the first row of the matrix, and the cells indicate the potential training needs of the target groups with respect to the considered ICT for manufacturing issues. If the individuals in the group have different capabilities regarding the ICT issues in manufacturing topics, then the "skills matrix" should be applied at the level of individuals.

\subsection{Step 6: Identification of trainers}

The aim of this step is to identify the experts on ICT for manufacturing that would provide the training material in connection with their field of expertise to the target audiences on the selected learning topics.

The selected trainers should have the necessary competencies on the use of ICT tools in the training activities and be up-to-date with the cutting-edge ICT matters in manufacturing related to the selected topics in order integrate them in these topics.

In the case of ActionPlanT project, the trainers are primarily selected among the partners of the ActionPlanT project on the basis of their expertise with respect to the topics retained for the IL actions. Only in the case where no expert from the consortium can provide the training for a given learning topic, then the appeal for experts from outside the consortium is considered.

\subsection{Step 7: Definition of learning content}

The learning content should be tailored to the needs of target groups with respect to the selected topics. This can be the improvement of existing knowledge/skills about ICT for manufacturing or the development of completely new knowledge/skills.

To facilitate the elaboration of the content of the learning modules, the usual approach consists in dividing the modules into subjects, the subjects in sub-subjects until obtaining the elementary subjects: the units. The rule is that the decomposition continues until obtaining of the most elementary elements for which it is easy to define the contents. Indeed, it is easier to develop the content for the concise and precise subjects than for the general subjects which can involve numerous and varied types of information.

The development of learning content for the selected topics is the responsibility of the trainers identified in the previous step. 
6.8 Step 8: Definition of IL action type and delivery mechanisms

For the delivery of the training programs, various instruments are proposed in order to suit the different learning styles and constraints of trainees and the requirements of the learning topics.

The list of the delivery methods used for the different learning topics considered in the IL actions includes both traditional and recent methods such as onsite seminar / workshop / conference, synchronized/ non-synchronized virtual classroom, Internetbased training, webinar, serious games, workshops at future factory, etc.

A special attention is given to human oriented approaches, employing ICT tools to support human interaction with the "real" environment and application, and humanto-human interaction (e.g. collaborative environments, etc.).

\subsection{Step 9: Delivery of IL activities}

The implementation of IL occurs during the different IL actions which are defined by using the competence-based IL model.

\subsection{Evaluation of IL activities}

The evaluation determines to what extent the training provided through the ActionPlanT IL approach has responded to the training requirements of target audiences. Kirkpatrick's four levels of evaluation model (Kirkpatrick 1959) is very useful to handle these issues. In ActionPlanT, we focus on the following three levels: (i) reaction of learners: what they thought and felt about the training, (ii) learning: the resulting increase in knowledge or capability, and (iii) behaviour: extent of behaviour and capability improvement and implementation / application.

In addition to the assessment of the level of success achieved through the training program, the follow up and evaluation allow to determine what updates are needed for the knowledge content and the delivery mechanisms in order to ensure an efficient and effective life-long training of the target groups.

Due to the importance of the evaluation and the validation of the proposed approach, a whole section (section 7) is dedicated to this issue.

\section{Evaluation and validation of the ActionPlant IL approach}

A set of IL actions, in the form of what we called Industrial Learning Pilot Events (ILPE), have been used for the assessment of the effectiveness of the suggested learning approach and knowledge delivery mechanisms. The evaluation output helped us to further improve the implementation aspects of the suggested approach and identify best practices in the use of knowledge delivery mechanisms for IL.

IL actions involved different S\&T themes on "ICT for manufacturing" and knowledge/training delivery mechanisms (Table 1). They were structured around a theoretical session for basic knowledge transfer and a practical session for hands-on exercise. Target groups included heterogeneous teams of engineers coming from industry and academia. 
IL actions in the realized ILPEs have been assessed against the achievement of a set of goals, related with: attitude, knowledge, skills, competencies (Tables 2, 3). The evaluation was based on the feedback of the trainees to a questionnaire, which is filled-in by all IL actions' participants/trainees, right after the end of the event. The questionnaire itself was structured in a way to assess the improvement of the attitude, knowledge, skills and competence of the trainees with respect to the introduced learning module.

The evaluation of the IL actions included both a qualitative and a quantitative assessment. Qualitative assessment aims to draw conclusions, on the basis of the statistical analysis of the trainees' feedbacks, about:

- the improvement of the attitude, knowledge, skills and competence of the trainees with respect to the introduced learning module,

- the actual work flow and performance of the group, the difficulties encountered by the trainees, their actual involvement and co-operation level,

- the strong / weak aspects of the introduced training delivery mechanism and areas of possible improvement for the training delivery.

A systematic approach was also being suggested for the quantified impact measurement of the IL actions and tested through the realized series of ILPEs as mentioned before. This approach is based on the concept of the weighted sum model (WSM), which is the best known and simplest multi-criteria decision analysis method (Fishburn 1967). The overall performance of an ILPE is calculated by taking the weighted sum of the normalized values of the ILPE performance criteria (building blocks of the learning process), i.e. attitude improvement, knowledge delivery, skills delivery and competences development (Equation 1). The weights assigned to these performance values depend on the relative importance of the respective performance criteria for each ILPE. For example, an ILPE may be focused more on practical training, e.g. skills delivery and competence development, rather than on theoretical aspects. Thus, higher weights would be assigned to the respective performance values.

A set of performance indicators is identified referring to each ILPE performance criterion (building block of the learning process). The weighted sum of the normalized values of these indicators is used to calculate the overall value of the respective ILPE performance criterion (e.g. Equation 2 is used for Attitude). The weights assigned to these values depend on the relative importance of the respective indicator in the achievement of the ILPE goals for the specific ILPE performance criterion. Each performance indicator is associated with a specific question included in the Questionnaire used for IL actions evaluation. The trainees' responses will be

Table 1 Initial set of IL actions

\begin{tabular}{lll}
\hline A\# & S\&T Theme & Training delivery mechanism \\
\hline 1 & Shop floor data processing & Teaching Factory \\
2 & Lean Manufacturing & Serious Game \\
3 & Closed Loop PLM & Best Practice Tutorial \\
\hline
\end{tabular}


Table 2 General goals of the IL actions

IL actions general goals

\begin{tabular}{|c|c|}
\hline Attitude & Create awareness, attract interest, increase motivation to learn \& apply \\
\hline Knowledge & $\begin{array}{l}\text { Create a basic technology understanding (basics of relevant theory \& software) } \\
\text { oriented to industrial practice, and acquaint with relevant ICT tools to search } \\
\text { for further information }\end{array}$ \\
\hline Skills & $\begin{array}{l}\text { Acquaint with the use of dedicated software tools, complete a well defined } \\
\text { task involving processing of data with the given tools }\end{array}$ \\
\hline Competencies & $\begin{array}{l}\text { Build-up basic ability to combine different pieces of knowledge, developed skills } \\
\text { and own understanding, to make decisions and address real life-like use cases }\end{array}$ \\
\hline
\end{tabular}

processed appropriately, so as to assign specific values to the performance indicators (Fig. 6).

For each ILPE performance criterion (building block of the learning process), the performance indicators help measuring in a quantified way the achievement of the respective training goal (Table 3). The performance indicators aim to capture the "contribution" of the IL actions in improving the attitude, knowledge, skills and competence of the trainees with respect to the introduced learning module. Thus, they measure the "difference" in the levels of attitude, knowledge, skills and competence, before and after the IL action as perceived by the trainees.

$$
P=w_{A} \times A+w_{K} \times K+w_{S} x S+w_{C} \times C
$$

$\mathrm{P}$

overall IL Action Performance Value

A, K, S, C overall value of the respective ILPE performance criterion

(attitude, knowledge, skills, competences)

Table 3 Example of specific goals of an IL action (Lean Manufacturing)

2nd IL action specific goals

\begin{tabular}{ll}
\hline Attitude & $\begin{array}{l}\text { Create awareness and attract interest with respect to Lean Manufacturing } \\
\text { and the supporting ICTs }\end{array}$ \\
Knowledge & Create a basic understanding about the major principles, pillars and limitations \\
of Lean Manufacturing, as well as about the manufacturing ICTs (e.g. MES, \\
ERP, RFID etc.) implementing the underlying principles and enabling \\
lean production \\
Apply different schemes for team work organization and information processing \\
in assembly operations, including traditional schemes, self-organization \\
and lean principles \\
Develop the capability of addressing realistic use cases involved in car assembly \\
operations, requiring decision making and optimization of teamwork organization \\
and information processing
\end{tabular}




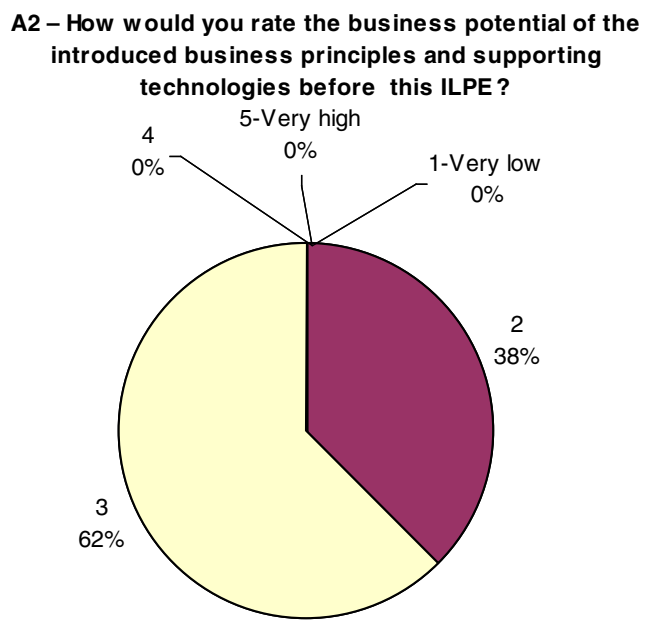

Mean rate before IL action: 2,625

A2 - How would you rate the business potential of the introduced business principles and supporting technologies after this ILPE?

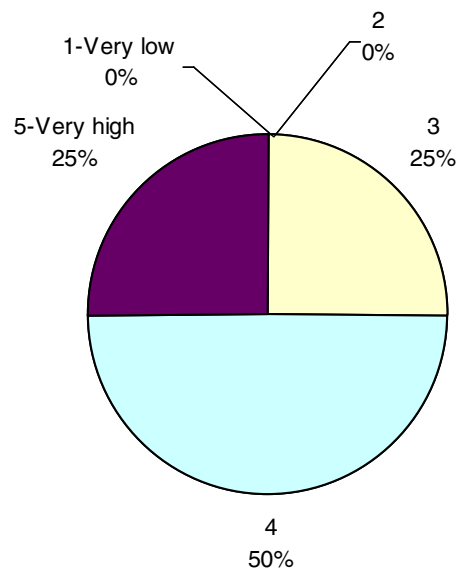

Mean rate after IL action: 4,00

Fig. 6 Example of performance indicator estimation

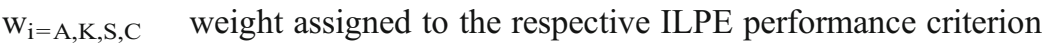
(IL action specific), $\Sigma \mathrm{w}_{\mathrm{i}}=1$

$$
A=w_{P I A 1} \times P I A_{1}+w_{P I A 2} \times P I A_{2}+\ldots+w_{P I A n} \times P I A_{n}
$$

A overall Attitude Performance Value

$\mathrm{PIAi}=1, \ldots, \mathrm{n} \quad$ performance indicators of Attitude (A)

wi $=\mathrm{PIA}_{1}, \ldots$, weight assigned to the respective performance indicator (IL action / $\mathrm{PIA}_{\mathrm{n}}$ technology specific), $\Sigma w i=1$ 


\section{Recommendations for ActionPlant IL approach}

In order to make the ActionPlanT IL model and the related concepts adopted by a large number of IL stakeholders, there is a need for actions and decisions to be taken by different actors involved in IL. Based on the lessons learned from ActionPlanT and the extensive review of related state-of-the-art, as an important part of the results of the project, we formulated a number of recommendations that can help achieving these goals.

The proposed recommendations (Tables 4, 5, 6, 7) are partitioned into four groups according to whether they relate to one of the 3 blocks of the ActionPlanT IL model: competencies specification, competencies implementation, and competencies development or to promotion and raising awareness about the importance of IL.

The first recommendation relates to the mechanism for defining e-skills for manufacturing and the second recommendation relates to the content developed using the mechanism in recommendation 1 . Recommendation 3 concerns the update of both the mechanism and the content.

Recommendation 4 looks at new ways of identifying training needs including those related to new professional needs triggered e.g., by business modernization, enlargement, etc. and recommendation 5 deals with the categorization of training needs according to the type companies.

Recommendation 6 considers the appropriate mechanisms to deliver e-skills for manufacturing workers and recommendation 7 relates to the definition of curricula using "cutting edge" related ICT.

Recommendation 8 deals with the promotion of IL as a means for developing ICT for manufacturing skills and recommendation 9 relates to involving public authorities and politicians in raising awareness on the importance of IL about ICT for manufacturing.

Table 4 Recommendations related to competencies specification block of ActionPlanT IL model

Recommendation Description

Recommendation 1:

A reference framework for e-skills in manufacturing

Recommendation 2:

List of new manufacturing e-skills

Recommendation 3:

Observatory for identifying emerging ICT for manufacturing skills
There is a need for a reference framework for e-skills in manufacturing. Such a framework should be the main reference for all IL stakeholders when dealing with issues related to e-skills in manufacturing. Since ICT progresses fast, the framework should be updateable to allow for the addition of new e-skills that may result from new developments in ICT for manufacturing.

There is a need for new manufacturing e-skills relating to advanced ICT for manufacturing. Such e-skills will allow manufacturing companies to implement and apply advanced ICT which are needed to boost competitiveness and innovation.

There is a need for an observatory for a continuous follow-up of the main trends and innovations in ICT for manufacturing, identifying the related ICT for manufacturing skills, and communicating the information to the IL stakeholders. 
Table 5 Recommendations related to competencies implementation block of ActionPlanT IL model

\begin{tabular}{ll}
\hline Recommendation & Description \\
\hline $\begin{array}{l}\text { Recommendation 4: } \\
\text { Go beyond traditional } \\
\text { training/learning needs analysis }\end{array}$ & $\begin{array}{l}\text { To be innovative, manufacturing companies should go } \\
\text { beyond the narrow traditional training/learning needs } \\
\text { analysis which focalize on the gaps in the current manufacturing } \\
\text { practices and look for new training/learning needs triggered by } \\
\text { new professional needs related to "cutting-edge"/ "fresh" ICT } \\
\text { developments. }\end{array}$ \\
$\begin{array}{l}\text { There is a need to elaborate on the relationship between the } \\
\text { type / size of a manufacturing company and the training } \\
\text { manking the type of } \\
\text { companies to the type of } \\
\text { chaining needs }\end{array}$ & $\begin{array}{l}\text { manufacturing skills. This will help manufacturing companies to } \\
\text { be aware of their training needs and support their decisions regarding } \\
\text { training actions. }\end{array}$ \\
\hline
\end{tabular}

The proposed recommendations to IL stakeholders address the different instruments, methodologies and activities, including raising awareness and motivation that can help them to effectively use the ActionPlanT IL model and the related concepts to deliver IL programs for the future e-skills in manufacturing. They have been validated or suggested by the experts that participated in the validation workshop which took place in Athens in May 2012.

\section{Summary and discussion}

The generic competence-based IL approach presented in this paper comprises: (i) an IL model which serves to represent and understand competence-based learning, and (ii) a methodology with a number of steps to implement IL in industrial organisations.

Table 6 Recommendations related to competencies development block of ActionPlanT IL model

\begin{tabular}{|c|c|}
\hline Recommendation & Description \\
\hline $\begin{array}{l}\text { Recommendation } 6 \text { : } \\
\text { Tailored delivery } \\
\text { mechanisms }\end{array}$ & $\begin{array}{l}\text { To deal with the various constraints facing the training/learning of manufacturing } \\
\text { workers and engineers such as time, mobility and ICT background and the } \\
\text { specificities of learning content about ICT for manufacturing, there is a need for } \\
\text { developing tailored delivery mechanisms to suit the different IL situations. A } \\
\text { repository establishing the relevance of the different delivery mechanisms to the } \\
\text { different IL situations can be an outcome of this action. At the extreme, } \\
\text { personalized delivery mechanisms (new fully-user tailored delivery } \\
\text { mechanisms) which are becoming more and more important for competencies } \\
\text { development can be considered. }\end{array}$ \\
\hline
\end{tabular}

Recommendation 7: To assist manufacturing companies in keeping up with the pace of rapid ICT

Curricula from
"cutting-edge" ICT development, there is a need to derive the learning content for IL curricula from recent achievements of research and innovation actions in the domain of ICT for manufacturing whose outcomes are potentially implementable in manufacturing. The content should be tailored to the current or future needs of manufacturing.. 
Table 7 Recommendations related to promotion and raising awareness about IL

Recommendation Description

Recommendation 8:

Promoting IL for developing ICT for manufacturing skills

Recommendation 9:

Involving public authorities and politicians
To make IL for developing ICT for manufacturing skills adopted by a significant number of manufacturing companies for a compelling impact on the skills levels in European manufacturing, there is a need for promotion through different actions and using various channels. These actions should emphasize the importance of e-Skills in manufacturing, highlight the links between e-Skills and innovation, encourage the collaboration between different stakeholders, provide the relevant information to concerned parties and offer incentives to manufacturing companies and especially SME.

Involving high-level non-industry related people in raising awareness about the importance of ICT for manufacturing IL can increase the impact of IL promotion campaigns and actions, and help motivate the IL stakeholders.

The ActionPlanT IL model is competence-based and is suitable for creating new knowledge assets related to "cutting edge" ICTs for manufacturing, identifying corresponding new professional competencies, and defining relevant learning actions to train workers to develop these competencies.

The ActionPlanT IL methodology has been developed on the of extensive state-of-the-art and gap analyses in order to propose a comprehensive methodology incorporating the most promising techniques including the emerging ones at each of its steps. The ActionPlanT IL methodology was developed in a way to overcome the weaknesses and fill the gaps of existing learning methodologies.

The novelty of the ActionPlanT IL methodology relates to two aspects: (i) the definition of IL actions using a competence-based IL model, and (ii) the comprehensiveness and the content of the steps of the methodology.

A metrics-based method has been developed to evaluate the implementation of ActionPlanT IL methodology. The metrics aim to capture the "contribution" of the methodology in improving the attitude, knowledge, skills and competence of the trainees with respect to the introduced learning topic.

During the implementation of each IL action, the suitability of the delivery mechanism to the learning topic has been tested and evaluated and the results were used to improve the choice of delivery mechanisms for learning topics in the forthcoming IL activities.

The results of the ActionPlanT activities have been evaluated by a group of 25 experts and their assessment is summarized in a set of recommendations for the development of ICT skills for manufacturing.

Among the future research issues related to the work presented in this paper, we can quote:

- development of mechanisms and methods to test the suitability of emerging delivery mechanisms to advanced ICT for manufacturing learning topics and learning constraints of the manufacturing labour force,

- definition of new ICT for manufacturing skills related to advanced ICT for manufacturing, 
- development of mechanisms and methods to define learning content for IL curricula from recent achievements of research and innovation actions in the domain of ICT for manufacturing.

Acknowledgments The research leading to these results has received funding from the European Community's Seventh Framework Programme (FP7/2007-2013) under grant agreement $n^{\circ} 258617$.

The work in the ActionPlanT project is a common effort among all its contributing partners: Agoria, EPFL, Fraunhofer IPK, Intercim, POLIMI, SAP, Tecnalia, University of Patras.

We would like also to thank the experts that participated in the validation workshop which took place in Athens in May 2012 for their contribution to the validation or suggestion of the recommendations.

\section{References}

Batista, M., Álvarez, M., Sánchez-Carrilero, M., Salguero, J., Marcos, M. (2009). CAL-CBT based virtual learning and training in machining engineering. A case study: CNC Lathe. In 2009 Materials Science Forum 625, pp. 19-28.

Brintrup, A. M., \& Ranasinghe, D. (2008). Organising industrial knowledge dissemination on frontier technology. European Journal of Engineering Education, 33(4), 471-481.

CEDEFOP (2009). Modernising vocational education and training: Fourth report on vocational training research in Europe: background report. CEDEFOP Reference Series. http://www.cedefop.europa.eu/ EN/publications/12892.aspx

Chen, J. C., \& Chen, J. (2004). Testing a new approach for learning teamwork knowledge and skills in technical education. Journal of Industrial Technology, 20(2), 37-46.

Chi, X., Speeding, T.A. (2007). A Web-based Intelligent Virtual Learning Environment for Industrial Continuous Improvement. In 2006 IEEE International Conference on Industrial Informatics, INDIN'06. art. no. 4053544, pp. 1102-1107.

Chryssolouris, G. (2005). Manufacturing systems - Theory and practice (2nd ed.). New York: Springer-Verlag.

Chryssolouris, G. (2006). Education for next generation manufacturing. Keynote Paper in IMS Vision Forum 2006. 12-13 April 2006, Seoul, Korea.

Chryssolouris, G., Mavrikios, D. (2005). A novel initiative for the Manufacturing Enterprises and Educational Institutions of the future: The Teaching Factory, Working Paper

Chryssolouris, G., Mourtzis, D. (2008). Challenges For Manufacturing Education. In Proceedings of CIMEC 2008, CIRP International Manufacturing Engineering Education Conference. Nantes, France, October 2008

Chryssolouris, G., Mavrikios, D., Fragos, D., Karabatsou, V., \& Pistiolis, K. (2002). A novel virtual experimentation approach to planning and training for manufacturing processes. International Journal of Computer Integrated Manufacturing, 15(3), 214-221.

Chryssolouris, G., Mavrikios D., Papakostas N., Mourtzis, D. (2006). Education in Manufacturing Technology \& Science: A view on Future Challenges \& Goals. Inaugural Keynote in Proceedings of the International Conference on Manufacturing Science and Technology (ICOMAST 2006). Melaka, Malaysia, August 2006.

Ellstrom, P.-E., \& Kock, H. (2009). Competence development in the workplace: concepts, strategies and effects. In K. Illeris (Ed.), International perspectives on competence development. Routledge: Developing Skills and Capabilities.

Fishburn, P. C. (1967). Additive Utilities with Incomplete Product Set: Applications to Priorities and Assignments. Baltimore: Operations Research Society of America (ORSA).

Folstad, A. (2008). Living Labs for innovation and development of information and communication technology: A literature review. Electronic Journal for Virtual Organizations and Networks 10, Special Issue on Living Labs.

Fürbringer, J.-M. (2010). Competence Based Education Project, Insttitute of Mecanical Engineering, Personal Communication. EPFL.

Hamade, R. F., Artail, H. A., \& Jaber, M. Y. (2011). A study of the impact of the willingness-to-learn of $\mathrm{CAD}$ novice users on their competence development. Computers and Industrial Engineering, 61(3), 709-720. 
Hauge, J.B., Duin, H., Oliveira, M., Thoben, K.D. (2006). User Requirements Analysis for Educational Games in Manufacturing. In Proceedings of the 12th International Conference on Concurrent Enterprising, p.8. Milan, Italy, June 2006.

IMS2020 deliverable: D2.3b Action Roadmap on KAT5-Innovation, competence development and education. Download link: http://data.fir.de/projektseiten/ims2020/files/IMS2020_Action-Roadmap_ KAT5.pdf

Kirkpatrick, D. L. (1959). Techniques for evaluating training programs. Journal of American Society of Training Directors, 13(3), 21-26.

Kramer, B.A., Azadivar, F. (2002). Manufacturing Learning Center: A model to enhance manufacturing engineering education. Technical Paper, ED02-276, Society of Manufacturing Engineers.

Lamancusa, J. L., Jorgensen, J., \& Zayas-Castro, J. (2001). The learning factory: a new approach to integrating design and manufacturing in engineering curricula. Journal of Engineering Education, 86, 103-112.

Manufuture High Level Group and Implementation Support Group (2006). ManuFuture PlatformStrategic Research Agenda, assuring the future of manufacturing in Europe, available online at: http://www.manufuture.org/manufacturing/?page_id=10

Marquardt, N., Robelski, S., \& Jenkins, G. G. (2011). Designing and evaluating a crew resource management training for manufacturing industries. Human Factors and Ergonomics In Manufacturing, 21(3), 287-304.

Matta, A. Shaw, A., Rabe, L., Bufardi, A., Folan, P., Moseng, B. (2007). Deriving specifications for training courses in large engineering integrated projects: A practical experience. In Joint SEFI-IGIP 2007 Annual Conference. Miskolc, Hungary

Mavrikios, D., Karabatsou, V., Fragos, D., \& Chryssolouris, G. (2005). A prototype virtual reality based demonstrator for immersive and interactive simulation of welding processes. International Journal of Computer Integrated Manufacturing, 19(3), 294-300.

Mayes, T., de Freitas, S. (2005). Review of e-learning theories, frameworks and models. JISC e-Learning Models Desk Study-Stage 2. http://www.elearning.ac.uk/resources/modelsdeskreview/;

Mirehei, S.M. (2009). Design and development of a simulation game for lean administrative process training. Master thesis, The University of Texas at San Antonio, UMI Number: 1464009.

O’Sullivan, D., Rolstadås, A., \& Filos, E. (2009). Global education in manufacturing strategy. Journal of Intelligent Manufacturing. doi:10.1007/s10845-009-0326-2.

OECD (2004). Lifelong Learning. Policy Brief. http://www.oecd.org/dataoecd/17/11/29478789.pdf

Paci, A.M., Lalle, C. (2008). Teaching factory for new education in competitive sustainable manufacturing. In Proceedings of DET2008 5th International Conference on Digital Enterprise Technology. Nantes, France, October 2008.

Rolstadås, A., \& Dolinšek, S. (2006). Global education in manufacturing: basic framework, industrial survey and possible implementation. International Research Journal on Managing Global Transition, 4(3), 261-278.

Rolstadås, A., Hussein, B. (2002). Hybrid Learning in Project Management-Potentials and Challenges, In PMI Conference. Seattle, USA, July 2002.

Schaf, F. M., Mueller, D., Bruns, F. W., Pereira, C. E., \& Erbe, H.-H. (2009). Collaborative learning and engineering workspaces. Annual Reviews in Control, 33, 246-252.

SME, 2010. Training Programmes. http://www.sme.org/cgi-bin/getsmepg.pl?/gmn/data/events/corptrain/ training.htm\&\&\&SME\&

Westkämper, E. (2008). The potential of regional manufacturing networks in Europe. St. Etienne: Presentation at Manufuture Conference.

Wong, S.F., Yang, Z.X., Cao, N., Ho, W.I. (2010). Applied RFID and virtual reality technology in professional training system for manufacturing. In IEEM2010-IEEE International Conference on Industrial Engineering and Engineering Management. art. no. 5674534, pp. 676-680.

Yaldiz, S. (2010). Virtual Training Centre for CNC: An Accomplished Cooperation Case. In the 5th International Conference on Virtual Learning ICVL 2010, pp. 253-259.

Zimmermann, M. (2010). On demand learning in manufacturing processes: Implementation by integrated multimedia streaming services. In 6th Advanced International Conference on Telecommunications, AICT 2010. art. no. 5489871, pp. 106-111 